\title{
Avaliação de preensão palmar em pacientes Pós-Covid-19 com disfunção em membro superior e seu impacto na funcionalidade
}

\section{An evaluation of handgrip strength in post-COVID-19 patients with upper limb dysfunction and its impact on functionality}

\author{
Carmine Thomas ${ }^{1 *}$, Patrícia de Morais Ferreira Brandão ${ }^{1}$, Paulo Henrique Muleta Andrade ${ }^{2}$, \\ Adriana Maria de Oliveira ${ }^{2}$, Mauricio Rodrigues Comin ${ }^{1}$
}

\begin{abstract}
RESUMO
O vírus SARS-CoV-2 compõe um grupo de vírus capazes de causar infecções respiratórias, que podem acarretar condições clínicas leves ou graves. O paciente pós-COVID-19 pode manter alguns sintomas como fadiga, falta de ar, fraqueza muscular, limitações nas Atividades de Vida Diária (AVDs), distúrbios emocionais e cognitivos, mesmo após terem recebido alta hospitalar. O objetivo deste estudo foi identificar questões relacionadas a sequelas neuromotoras, bem como mensurar a força de preensão palmar e analisar a funcionalidade e desempenho ocupacional de pessoas acometidas pela COVID-19 submetidas a reabilitação em um Centro Especializado em Reabilitação. Trata-se de um estudo do tipo observacional, transversal e com abordagem quantitativa de dados. Os participantes foram submetidos a testes de força de preensão palmar, além da aplicação de escalas que mensuram a funcionalidade dos membros superiores e a qualidade de vida. Os resultados apontam que lesões no membro superior de pacientes com sequelas pós COVID-19 interferem na realização das AVDs, principalmente aquelas relacionadas a alimentação e higiene. A neuropatia sensorial é a queixa principal, mostrando-se com muita intensidade, principalmente naqueles que passaram por longos períodos de internação. Conclui-se que tais condições dificultam as ocupações, principalmente no autocuidado e nas tarefas domésticas e do trabalho. Assim, é importante ressaltar que o conhecimento sobre a COVID-19, seus sinais e sintomas, bem como as possiblidades de tratamento, são de suma importância para que a pessoa não se prive de sua ocupação humana.
\end{abstract}

Palavras-chave: Coronavírus; Força da Mão; Membros Superiores;

\section{ABSTRACT}

The SARS-CoV-2 virus composes a group of coronaviruses capable of causing respiratory infections, which may lead to mild or severe clinic conditions. Post-COVID-19 patients may keep presenting some symptoms such as fatigue, shortness of breath, muscle weakness, limitations to Activities of Daily Living (ADLs), emotional and cognitive disorders, even after being discharged from the hospital. The objective of this study was to identify issues related to neuromotor sequelae, as well as to measure the handgrip strength and analyze the functionality and occupational performance of the people affected by COVID-19 who underwent rehabilitation in a Center Specialized in Rehabilitation, correlating the data with the performance of ADLs. This is an observational, cross-sectional study with a quantitative data approach. The participants were submitted to handgrip strength tests, besides the application of scales that measured the functionality of the upper limbs and quality of life. The results point out that upper limb injuries in patients with post-

\footnotetext{
${ }^{1}$ Universidade Federal de Mato Grosso do Sul (UFMS)

*E-mail: carminethomas97@ gmail.com

${ }^{2}$ Centro Especializado em Reabilitação da Associação de Pais e Amigos dos Excepcionais de Campo Grande - Mato Grosso do Sul: CER APAE
} 
COVID-19 sequelae interfere with the performance of ADLs, especially those related to alimentation and hygiene. Sensory neuropathy is the main complaint, showing up with great intensity - especially in those who have been hospitalized for long periods - and affecting their occupational performance. It is concluded that such conditions make occupations more difficult, especially in self-care and domestic and work-related tasks. Thus, it is important to emphasize that knowledge about COVID-19, its signs and symptoms, as well as the possibilities of treatment, are of utmost importance for the person not to be deprived of their human occupation.

Keywords: Coronavirus; Hand Strength; Upper Limbs;

\section{INTRODUÇÃO}

O cenário atual encontra-se diferente, tem-se um vírus acometendo os seres humanos de forma grave e mundial. Muitos vão a óbito e os que sobrevivem apresentam sequelas as quais, por vezes, acometem a funcionalidade e autonomia do indivíduo, deixando-o dependente de outros para realizar suas Atividade Básicas de Vida Diária (AVDs).

O SARS-CoV-2 compõe um grupo de vírus capazes de ocasionar infecções respiratórias, com repercussões clínicas leves ou graves. A primeira identificação desse vírus em seres humanos foi em 1937, entretanto, somente em 1965 foi determinado como coronavírus, por apresentar um perfil microscópico parecido a uma coroa (SILVA et al, 2021). O avanço dos casos no mundo tem sido observado desde o planejamento de intervenções para respostas rápidas à disseminação da doença, e nesse contexto é possível incluir ações de detecção precoce, isolamento, vigilância epidemiológica, medidas de prevenção e controle e a avaliação de impactos sanitários (LAKE et al, 2020). De acordo com pesquisadores, a transmissão está ligada às diversas formas de contágio, por exemplo: contato direto com gotículas de saliva, aerossol, fezes e urina e pelo contato da mucosa com partículas nas superfícies pelas mãos, ou seja, é um alto risco de disseminação para as pessoas (SILVA et al, 2021).

Os pacientes pós-COVID-19, mesmo após terem recebido alta hospitalar, podem manter alguns sintomas, desde falta de ar, tosse, fadiga, fraqueza muscular e limitações nas atividades diárias, distúrbios emocionais e cognitivos associados a diversas complicações. Sendo assim, percebe-se que o envolvimento multissistêmico do vírus SARS-CoV-2 pode expressar formas graves da doença relacionadas a fatores de risco, portanto, a reabilitação integral deve ser contínua, com diretrizes de tratamento para 
restaurar a condição física, psicológica e social do paciente, a fim de promover maior qualidade de vida e independência (PIEDRA et al, 2020).

O comprometimento motor dos sujeitos pós-COVID-19 pode prejudicar a funcionalidade e a capacidade de realizar atividades de vida diária, alterar o desempenho profissional, dificultar a interação social e causar sedentarismo, aumentando o risco de comorbidades (SANTANA et al, 2021).

De acordo com Zlotnick et al (2019), a exploração limitada do valor das ocupações, principalmente no contexto atual, acarreta o desengajamento na vida ocupacional, com prejuízos visíveis à pessoa adoecida e aos seus familiares e/ou cuidadores.

Diante do exposto, o objetivo do trabalho foi identificar questões relacionadas às sequelas neuro motoras, bem como traçar o perfil da força de preensão palmar e analisar a funcionalidade e desempenho ocupacional dos participantes acometidos pelo COVID19 submetidos a reabilitação em um Centro Especializado em Reabilitação (CER IV).

\section{MÉTODOS}

Trata-se de um estudo do tipo observacional, transversal e com abordagem quantitativa de dados. Para a análise estatística, inicialmente foi aplicado o teste de Shapiro-Wilk para verificar se os dados apresentaram características paramétricas ou nãoparamétricas. A análise identificou que as variáveis deste estudo não contemplaram os preceitos de normalidade da curva de Gauss e, portanto, os pesquisadores optaram pelo uso da estatística não-paramétrica. Todos os dados estão descritos em mediana \pm intervalo interquartil (variáveis contínuas), número de ventos e porcentagem (variáveis categóricas). Gráficos Box-plot foram incluídos para descrever precisamente algumas das variáveis contínuas estudadas. Testes estatísticos inferenciais foram aplicados para investigar o padrão dos resultados. O teste de qui-quadrado (com e sem tabelas cruzadas) foi aplicado para analisar variáveis categóricas. As variáveis contínuas foram analisadas pelos testes U Mann Whitney, Kruskrall-Wallis e Wilcoxon. Testes de correlação de Spearman foram realizadas para comparar associação entre DASH (Disahililies of lhe Arm, Shoulder and Hand) e variáveis mensuradas no equipamento de dinamometria. Para 
todas as análises, o intervalo de confiança foi estipulado em $95 \%$ e o nível de significância foi aceito em $5 \%(\mathrm{p}<0,05)$.

Para a obtenção dos objetivos propostos, no mês de dezembro de 2021, os participantes foram alocados em um único grupo formado por participantes em fase de reabilitação de sequelas pós COVID-19 que realizam acompanhamento em um ambulatório COVID-19 localizado no CER/APAE. Ressalta-se que o estudo foi previamente aprovado pelo Comitê de Ética em Pesquisa da Universidade Federal de Mato Grosso do Sul (UFMS), e todos os participantes da pesquisa estavam de acordo e assinaram o Termo de Consentimento Livre e Esclarecido (TCLE). Os critérios de inclusão foram pessoas com síndrome pós-COVID-19, com idade entre 30 e 59 anos completos, de ambos os sexos e que estivessem em acompanhamento no ambulatório da instituição.

Foram excluídas pessoas que apresentaram quadro de afasia e outras condições neurológicas, que impossibilitassem a realização das entrevistas e avaliações, declínios cognitivos que incapacitassem o sujeito de compreender os objetivos e testes dessa pesquisa ou influenciassem no consentimento do mesmo em participar da pesquisa e pessoas com histórico de outras patologias musculoesqueléticas ou lesões ortopédicas em membro(s) superior(es), o que poderia influenciar os resultados das avaliações, e aqueles que se recusaram a participar da pesquisa e/ou a assinar o TCLE.

Para realizar a pesquisa, foram utilizados dois instrumentos: o Disabililies of the Arm, Shoulder and Hand (DASH) e o KFORCE Grip. Além de uma avaliação inicial com um questionário composto por algumas perguntas pessoais básicas, como: idade, sexo, tempo de internação, níveis de dependência e independência ao realizar as atividades de vida diária. O instrumento DASH serve para avaliar a capacidade de realizar movimentos motores finos, bem como movimentos mais amplos e que exigem capacidades motoras, como força e destreza (CHENG, 2006). Cada item deve ser pontuado numa escala de um (01) a cinco (05) de acordo com o nível de esforço necessário para realizar a atividade descrita. Com o instrumento, também é possível mensurar a sintomatologia e avaliar como as desordens de membro superior podem impactar ou comprometer a funcionalidade e outros aspectos da vida da pessoa (CHENG, 2006). Os itens informam o grau de dificuldade no desempenho de atividades; a intensidade dos sintomas de dor, fraqueza, rigidez e parestesia; o comprometimento de atividades sociais; a dificuldade para dormir e o comprometimento emocional. 
O KFORCE Grip é utilizado para avaliar a força de preensão da mão, sendo possível avaliar a isométrica pela forma da força de pico, força média, parâmetros de velocidade, bem como fadiga. $\mathrm{O}$ dinamômetro Grip permite medir quantitativamente a fraqueza de preensão causada por lesão em comparação com a força da mão saudável, sendo equipado com transdutores de força eletrônicos e oferecendo biofeedback em tempo real através do aplicativo KFORCE. Ao final de cada avaliação, obtém-se um relatório completo, que pode ser gerado em PDF, comparando a força utilizada para realizar o movimento de preensão (KINVENT, 2021). Durante a avaliação da força da preensão palmar, foi utilizado o protocolo de três repetições durante cinco segundos, seguidos de 10 segundos de descanso.

\section{RESULTADOS}

Considerando-se o período de coleta de dados, houve a participação de 30 pessoas com diagnóstico de síndrome pós-COVID-19, sendo 11 homens (36,7\% da amostra) e 19 mulheres (63,3\% da amostra). A proporção de homens e mulheres neste estudo não apresentou diferença estatística $\left(X^{2}=2,133 ; p=0,144\right)$. A média de idade dos participantes foi de 49,0 7,5 anos, considerando a faixa etária adulta.

Em relação ao trabalho, apenas $10,0 \%$ dos participantes estavam ativos no trabalho e 90,0\% estavam afastados, ou seja, pacientes sequelados de COVID-19 encontram-se mais afastados do trabalho do que na atividade regular de sua profissão $\left(x^{2}=19,200 ; p=0,001\right)$.

Os participantes ficaram em média 19,47 $\pm 21,10$ dias internados. A distribuição dos dias de internação dos participantes encontra-se abaixo. 
Gráfico 1: Categorização de pacientes ativos ou afastados do trabalho.

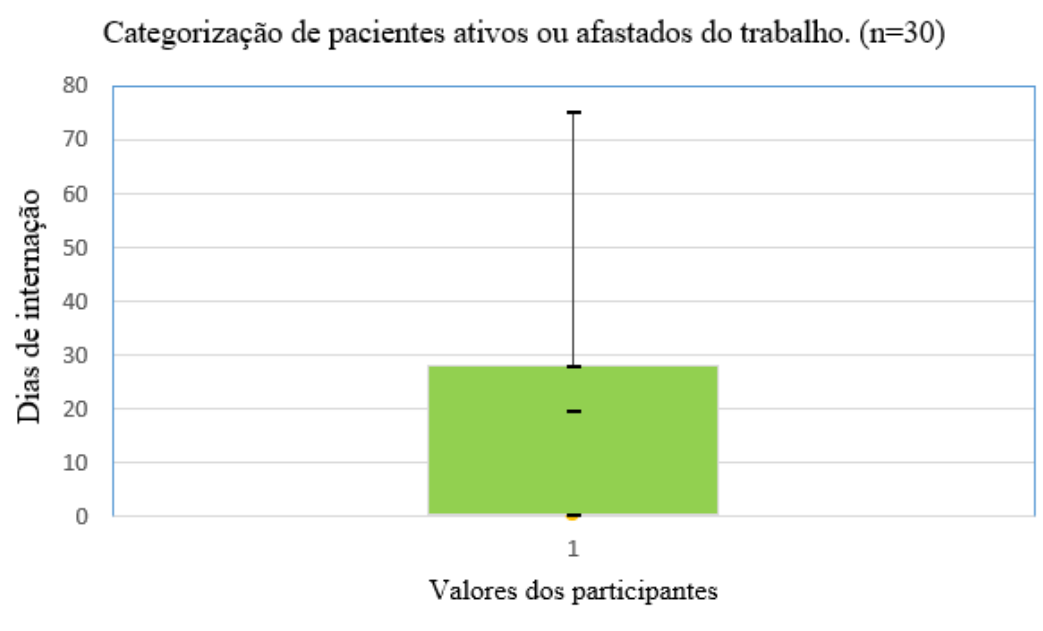

Fonte: Autora, 2022.

Os participantes que estão ativos no trabalho não precisaram ficar internados em hospital ( 0 dias de internação). Os participantes afastados do trabalho tiveram 14,0 $\pm 27,0$ dias internados (95\% dos afastados encontram-se entre 14,0 e 27,0 dias internados). Este aspecto constitui diferença significativa entre as pessoas que estão trabalhando e aquelas afastadas do trabalho (UMW=7,500; $\mathrm{p}=0,015)$.

A tabela a seguir mostra a queixa principal dos participantes. A análise estatística mostrou que a queixa principal dos participantes refere-se à neuropatia sensorial $\left(x^{2}=9,667 ; p=0,046\right)$.

Tabela 1: Queixa principal dos participantes.

\begin{tabular}{lccc}
\hline Queixa principal & Participantes (n) & Percentil (\%) & p \\
\hline Alteração Cognitiva & 1 & 3,3 & 0,046 \\
Dispnéia & 4 & 13,3 & \\
Dor ao realizar AVD & 6 & 20,0 & \\
Fraqueza muscular & 8 & 26,7 & \\
Neuropatia & 11 & 36,7 & \\
Total & 20 & 100,0 &
\end{tabular}

Fonte: Autora, 2022.

A tabela abaixo mostra o perfil dos participantes em relação a AVDs (alimentação, vestuário e higiene). A análise estatística apontou que a proporção de pessoas 
independentes, com necessidade de auxílio parcial e aquelas totalmente dependentes é semelhante para alimentação $(\chi 2=5,000 ; p=0,082)$, vestuário $(\chi 2=4,200 ; p=0,122)$ e higiene $\left(x^{2}=5,000 ; p=0,082\right)$.

Tabela 2: Nível de dependência nas AVDs.

\begin{tabular}{lcccc}
\hline $\begin{array}{l}\text { Atividades } \\
\text { funcionais }\end{array}$ & $\begin{array}{c}\text { Independente } \\
(\boldsymbol{\%})\end{array}$ & $\begin{array}{c}\text { Auxílio parcial } \\
(\boldsymbol{\%})\end{array}$ & $\begin{array}{c}\text { Dependente } \\
(\boldsymbol{\%})\end{array}$ & $\mathbf{p}$ \\
\hline AVDs: & & & & \\
Alimentação & 33,3 & 16,7 & 50,0 & 0,082 \\
Vestuário & 30,0 & 20,0 & 50,0 & 0,122 \\
Higiene & 33,3 & 16,7 & 50,0 & 0,082 \\
\hline
\end{tabular}

Fonte: Autora, 2022.

Em relação ao membro acometido, 33,3\% dos participantes tiveram afetado o membro superior direito, $13,3 \%$ o membro superior esquerdo, $30,0 \%$ tiveram ambos os membros afetados e 23,3\% não tiveram nenhum membro afetado. A proporção de pessoas em cada grupo de acometimento não constituiu diferença significativa $(\chi 2=2,800$; $\mathrm{p}=0,423)$. O acometimento do membro superior teve impacto principalmente na alimentação e na higiene dos participantes $(\chi 2=12,841 ; p=0,046$ para ambas as análises $)$.

Os participantes apresentaram 108,43 $\pm 16,91$ pontos no instrumento DASH. Os valores encontrados no DASH estão mais detalhados na figura abaixo, levando em consideração a pontuação de 01 a 05, de acordo com o nível de esforço necessário para realizar a atividade descrita.

Gráfico 2: Média de pontuação do instrumento DASH.

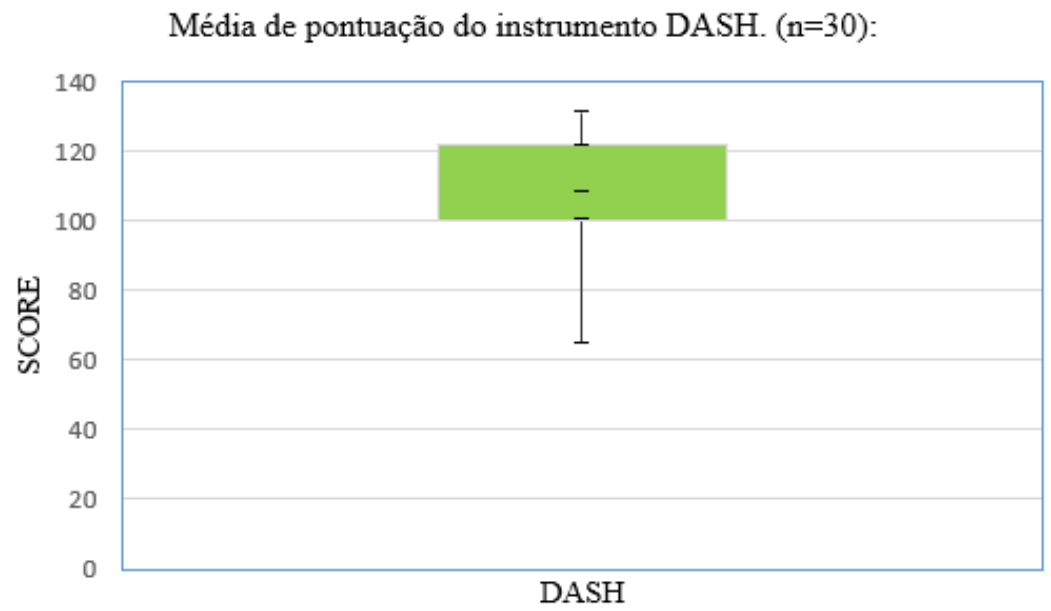

Fonte: Autora, 2022. 
A tabela abaixo demonstra a relação do DASH segundo pessoas independentes, aquelas que precisam de auxílio parcial e as dependentes nas AVDs. Ainda que se observe um aumento do DASH segundo o grau de dependência do participante, a comparação entre grupos não foi significativa $(\mathrm{KW}=1,896 ; \mathrm{p}=0,388)$.

Tabela 4: Relação Dash com níveis de independência, auxilio parcial e dependência.

\begin{tabular}{lcc}
\hline AVDs & DASH & p \\
\hline Independente & $58,5 \pm 33,2$ & 0,388 \\
Necessita de auxílio parcial para & $61,4 \pm 13,0$ & \\
realizar atividades específicas & & \\
Dependentes & $70,0 \pm 14,0$ &
\end{tabular}

Fonte: Autora, 2022.

O fato do paciente estar ativo ou afastado do trabalho não apresentou relação com DASH, força, potência e fadiga ( $p>0,05$ em todas as comparações) e o grau de dependência nas AVDs não teve relação com força, potência e fadiga ( $p>0,05$ em todas as comparações).

\section{DISCUSSÃO}

Levando-se em consideração a temática ainda recente, percebe-se que os sintomas e as possíveis consequências da COVID-19, mesmo entre aqueles que tiveram contato apenas com a forma branda da doença, vêm apresentando sequelas e limitações no dia a dia, interferindo assim, principalmente no desempenho ocupacional e na realização das atividades de vida diária (AVDs) e atividades instrumentais de vida diária (AIVDs).

De acordo com Wan et al, (2020), a incidência de COVID-19 não é influenciada pelo sexo. No entanto, em alguns países, a incidência do vírus é significativamente maior entre os homens do que entre as mulheres (MO et al, 2020).

De acordo com Betron et al (2020), existem alguns fatores que podem contruibuir para o aumento da incidência de COVID-19 entre homens, tais como: taxa de tabagismo, doenças respiratórias e cardíacas, sistema imunológico e hormônios sexuais masculinos mais fracos (EJAZ et al, 2021). 
De acordo com Souza et al (2020), o diferencial por sexo, analisado por meio da razão entre as taxas específicas de mortalidade, é crescente com a idade, sendo o risco de morte dos homens cerca de duas vezes o das mulheres nos grupos de 50 a 59 e 70 a 79 anos. Entretanto, de acordo com Almeida et al (2021), os casos de COVID-19 em trabalhadores do sexo feminino representam a maioria na amostra da pesquisa, superando em 19\% a proporção de casos registrados na Bahia para o sexo feminino. Contudo, o que pode indicar maior vulnerabilidade para o adoecimento por COVID-19 pelas trabalhadoras é devido à feminilização da força de trabalho e à divisão sexual do trabalho no setor saúde (Teixeira et al, 2020; Duarte et al, 2020).

No que diz respeito ao tempo de internação, não há evidências sobre as diferenças entre os sexos, porém há entre as idades, permanecendo por mais tempo hospitalizados aqueles com mais de 45 anos (Wang et al, 2020). No presente estudo, observa-se uma amostra de participantes com idade média de 49 anos, sendo essa a média que permaneceu hospitalizado por um período maior.

Sabe-se que a COVID-19, em alguns casos, representa uma série de sintomas e complicações prolongadas, que vão além do período inicial da doença ou que aparecem no período de pós-infecção (AGUIAR et al, 2021). Estas sequelas têm potencial de comprometer as atividades diárias e prejudicar a saúde do trabalhador (Xiong et al, 2021). Assim, é de suma importância observar essas questões e promover ações para amenizar esse impacto no cotidiano das pessoas acometidas pelo virus (AGUIAR et al, 2021).

A presente amostra aponta que apenas $10,0 \%$ dos participantes estavam ativos no trabalho e 90,0\% estavam afastados, ou seja, pacientes com sequelas de COVID-19 encontram-se mais afastados do trabalho do que na atividade regular de sua profissão, causando uma ruptura em seu cotidiano e gerando um desempenho insatisfatório das atividades de vida diária. Além dessas sequelas causarem impacto econômico para as famílias, elas também podem causar impacto psicológico, fazendo com que o trabalhador muitas vezes se sinta incapaz de voltar a trabalhar e de realizar suas atividades normalmente.

De acordo com um estudo realizado, levando em consideração 60 dias após o início dos sintomas da COVID-19, entre 143 pacientes, somente 12,6\% afirmaram não apresentar nenhum sintoma da doença após a infecção, o restante ainda apresentava sintomas, com relatos de piora da qualidade de vida entre $44,1 \%$ dos pacientes (AGUIAR et al, 2021). No presente estudo, todos os participantes relataram ainda apresentar 
sintomas, mesmo após um longo período posterior à infecção. Diante disso, observa-se uma interferência na realização das atividades de vida diária, diminuindo assim a qualidade de vida e a capacidade de trabalho dos participantes (AGUIAR et al, 2021). Evidenciou-se em outro estudo que, entre 177 participantes, os sintomas persistentes da COVID-19 foram relatados por 26,6\% dos pacientes com idade entre 18 e 39 anos, 30,1\% entre 40 a 64 anos e 43,3\% com 65 anos ou mais, e os acometidos relataram apresentar dificuldade em pelo menos uma atividade de vida diária, bem como uma baixa produtividade (AGUIAR et al, 2021).

Os dados apontam que a maior parte da população ativa no mercado de trabalho, com faixa etária de 18 a 64 anos, está suscetível a alguma condição clínica pós-COVID, que acarreta um desempenho ocupacional insatisfatório (AGUIAR et al, 2021). Outra pesquisa realizada acompanhou um corte de 177 indivíduos por até nove meses após os primeiros sintomas da doença. Aproximadamente 30\% deles relataram sintomas persistentes, não retornando ao seu estado normal de saúde e apresentando também dificuldades em seu retorno ao trabalho (Xiong et al, 2021). Diante disto, percebe-se um grande impacto na saúde do trabalhador, evidenciando-se o quanto as sequelas da COVID-19 podem gerar agravos na saúde, tanto física quanto mental, prejudicando também o trabalho e a geração de renda desses indivíduos.

Em relação às sequelas apresentadas, o presente estudo identificou que, dos 30 participantes, 11 relataram a neuropatia como queixa principal, seguida de fraqueza muscular e dificuldades em realizar as AVDs, tendo em vista que os pacientes que ficaram por mais tempo hospitalizados apresentaram mais sequelas, ficando dependentes de auxílio parcial ou total para a realização as atividades de vida diária.

Em um estudo realizado na Itália, 143 pacientes foram avaliados dois meses depois de terem alta e obtiveram o seguinte resultado: apenas 18 participantes não apresentavam qualquer sintoma relacionado ao coronavírus, enquanto $87,4 \%$ relataram persistência de pelo menos um sintoma, entre eles fadiga $(53,1 \%)$ e falta de ar $(43,4 \%)$ (CARFI et al, 2020). De acordo com estudos publicados, estima-se que doentes com condições clínicas mais graves, especialmente os que passaram por grandes periodos de internação, bem como de ventilação mecânica, apresentam mais complicações a longo prazo e dificuldades na recuperação (LUZ et al, 2021). Segundo Carvalho-Schneider et $a l$, (2020), pelo menos 68\% das pessoas com COVID-19 ainda mantinham um sintoma até os primeiros 30 dias pós- infecção e $66 \%$ continuavam a apresentar sintomas 60 dias 
após a infecção, ou seja, a maioria das pessoas acometidas ainda apresenta sintomas mesmo após o período de infecção.

Entre os principais sintomas apresentados, a fraqueza muscular, o descondicionamento, as miopatias e neuropatias podem ser resultados secundários da COVID-19, levando, potencialmente, a um aumento da incapacidade e mortalidade (BRAZÃO et al, 2021). De acordo com Sun et al (2020), 50\% dos pacientes internados na UTI (Unidade de Terapia Intensiva) com síndrome do desconforto respiratório agudo (SDRA) apresentaram polineuropatia crítica associada.

Corroborando com o presente estudo, Finsterer et al (2021) apresenta alguns fatores existentes associados a neuropatia, são eles: diabetes pré-existente, obesidade, uso de drogas, permanência prolongada na UTI, bem como o uso de drogas utilizadas para o tratamento de sintomas da COVID-19. Outro dado importante a ser levado em consideração é que pacientes que requerem posição de pronação para tratamento da SDRA na UTI por conta do vírus, estão predispostos para desenvolver neuropatia de compressão (FINSTERER et al, 2021). Acredita-se que danos pré-existentes de nervos periféricos, efeitos colaterais dos medicamentos usados para tratar as manifestações da COVID-19 e o posicionamento dos pacientes parecem ser as causas mais relevantes da neuropatia após a contaminação do coronavírus.

Dessa forma, por ser uma neuropatia que causa prejuízo no desempenho ocupacional e na qualidade de vida, faz-se necessário o uso de alguns elementos na prática como forma de avaliar, prevenir e/ou reabilitar, a fim de amenizar prejuízos futuros.

Em relação aos níveis de dependência, o presente estudo identifica que a maioria dos participantes encontrava-se dependente para a realização das AVDs, entretanto, os participantes que tiveram o membro superior acometido relataram sentir mais dificuldade principalmente na alimentação e na higiene, o que corrobora com o estudo desenvolvido em um Hospital público no Rio Grande do Sul, onde os participantes foram separados em quatro grupos, de acordo com o grau de dependência e, desses, 32 apresentaram um maior grau de dependência para a realização das AVDs, necessitando de auxílio parcial e/ou total para realizar as atividades (LOPES et al, 2021).

Um estudo constatou que, dos 65 participantes avaliados, $66,15 \%$ perceberam limitações funcionais, principalmente ao realizar as AVDs e, desses, 15,38\% relataram limitações de moderadas a grave, necessitando de auxílio parcial ou total para a realização das atividades cotidianas (AMARAL et al, 2021). Porto et al, 2021, realizou um estudo 
com 888 participantes, em que 170 deles responderam que necessitam de auxílio ou supervisão para as tarefas de alimentação (13,3\%), higiene pessoal (21\%), uso do banheiro (20\%), tomar banho (6,5\%), vestir-se (11\%), subir e descer escadas (16\%) e andar (12,4\%). Segundo Nery et al, 2020, em um estudo, 117 pessoas foram avaliadas por via telefônica, sendo questionadas acerca de sua capacidade funcional ao realizar as atividades de vida diária. A maioria dos pacientes avaliados apresentava grau de funcionalidade independente, entretanto, 25 indivíduos informaram que, em maior ou menor grau, a internação hospitalar pela contaminação do coronavírus impactou na sua capacidade funcional, quando comparado ao momento pré-doença.

Mesmo que alguns participantes relatassem não necessitar de auxílio para a realização das atividades, quando estes foram questionados sobre a realização de atividades mais específicas, todos apresentaram um grau de dependência, por menor que seja, do que previamente à doença. Diante disso, percebe-se o quão importante é a intervenção precoce de uma equipe multiprofissional, a fim de evitar impactos e agravos futuros.

Em relação a sequelas apresentadas nos membros superiores, 33,3\% dos participantes tiveram o membro superior direito afetado, 13,3\% o membro superior esquerdo, 30,0\% tiveram ambos os membros afetados e $23.3 \%$ não tiveram nenhum membro afetado. A queixa principal dos participantes que tiveram o(s) membro(s) superior(s) afetado(s) foi a neuropatia, relatando sentir dor, formigamento e dormência. De acordo com os resultados do presente estudo, evidencia-se que o acometimento do membro superior do participante não teve impacto direto no vestuário, porém apresentou importante impacto na alimentação e na higiene dos participantes. Tendo em vista esse resultado, na amostra percebeu-se que aqueles que relataram função manual alterada são mais dependentes para alimentação, vestuário e higiene, o que reforça que os impactos da condição pós-COVID possuem grande interferência no desempenho das atividades de vida diária.

Os partcipantes apresentaram uma média de força de 11,6 Kg no membro superior direito e $12,9 \mathrm{Kg}$ no membro superior esquerdo. De modo geral, as diferenças de força de mão domintante e mão não dominante não foram significativas. O efeito da força da mão dominante e não dominante também foi investigado por Armstrong et al (1999), que concluiu que as diferenças encontradas entre a força de preensão palmar da mão dominante e não-dominante de 83 pessoas, com idade entre 18 e 72 anos, são pequenas 
(em torno de 10\%). Dias et al (2010) encontrou diferenças da força em torno de $10 \%$ entre as mãos em um grupo de 50 mulheres e 50 homens. De modo geral, apesar das diferenças serem pequenas, a mão dominante apresenta melhor desempenho que a mão nãodominante em ambos os sexos.

Percebe-se que alguns resultados são diretamente proporcionais, ou seja, quanto maior a força apresentada pelo individuo, maior a potência e menor a fadiga. Entretanto, em comparação com o resultado da aplicação do instrumento DASH, constata-se que, quanto menor a força, menor a potência e maior a fadiga, sendo assim, observa-se que o indivíduo pode apresentar mais dependência parcial ou total para realizar as atividades de vida diária.

\section{CONCLUSÃO}

A realização da presente pesquisa possibilitou identificar e analisar as principais sequelas neuromotoras dos participantes acometidos pela COVID-19 e assim traçar o perfil desses indivíduos a fim de obter dados em relação a sequelas e à força da preensão palmar referente ao(s) membro(s) acometido(s). Conforme se percebe, a síndrome pósCOVID-19 compromete a funcionalidade e o desempenho ocupacional, principalmente em atividades envolvendo o membro superior. A principal queixa é a neuropatia sensorial, mostrando-se com muita intensidade, principalmente naqueles que passaram por longos períodos de internação, interferindo no desempenho das atividade de vida diária e na rotina. Além do acometimento físico, percebeu-se um acometimento emocional importante, devido a situações traumáticas vividas pelos participantes. Tais condições tem uma interferência nas ocupações, principalmente no autocuidado e na produtividade em relação às tarefas domésticas e do trabalho. Assim, é importante ressaltar que o conhecimento sobre a COVID-19, seus sinais e sintomas, bem como as possiblidades de tratamento, são de suma importância para que a pessoa não se prive de suas tarefas cotidianas, bem como de sua ocupação humana. Tendo em vista o fato da pandemia ainda se fazer presente não só no país, mas no mundo todo, esses achados são relevantes e demonstram a necessidade de ainda mantermos os cuidados necessários - a fim de reduzir o impacto da COVID-19 -, bem como continuar pesquisando sobre a COVID-19, suas sequelas e possíveis impactos na saúde humana. 


\section{REFERÊNCIAS}

AGUIAR, Bianca Fontana; SARQUIS, Leila Maria Mansano; MIRANDA, Fernanda Moura D.'Almeida. Sequelas da Covid-19: uma reflexão sobre os impactos na saúde do trabalhador. Research, Society and Development, v. 10, n. 14. 2021.

ALMEIDA, Suzana Mendes et al. Perfil epidemiológico dos casos de Covid-19 relacionados ao trabalho no estado da Bahia. Rev. baiana saúde pública, p. 93-108, 2021.

AMARAL, Kaueli Rodrigues et al. Impacto da covid-19 nas limitações funcionais de indivíduos em alta hospitalar. Anais do Salão Internacional de Ensino, Pesquisa e Extensão, v. 13, n. 1, 2021.

ARMSTRONG, C. A.; OLDHAM, Jackie A. A comparison of dominant and nondominant hand strengths. The Journal of Hand Surgery: British \& European Volume, v. 24, n. 4, p. 421-425, 1999.

BETRON, Myra et al. Men and COVID-19: Adding a gender lens. Global Public Health, v. 15, n. 7, p. 1090-1092, 2020. Disponível em:

<http://dx.doi.org/10.1080/17441692.2020.1769702>.

CARFÌ, Angelo et al. Persistent symptoms in patients after acute COVID-19. Jama, v. 324, n. 6, p. 603-605, 2020.

CARVALHO-SCHNEIDER, Claudia et al. Follow-up of adults with noncritical COVID-19 two months after symptom onset. Clinical Microbiology and Infection, v. 27, n. 2, p. 258-263, 2021.

DA LUZ BRAZÃO, Maria; NÓBREGA, Sofia. Complicações/Sequelas Pós-Infeção por SARS-CoV-2: Revisão da Literatura. Medicina Interna, v. 28, n. 2, p. 184-194, 2021.

DA SILVA, Hercilia Martins. Disabilities of the arm, shoulder, and hand-DASH: análise da estrutura fatorial da versão adaptada para o português. 2006.

DUARTE, Magda Machado Saraiva et al. Descrição dos casos hospitalizados pela COVID-19 em profissionais de saúde nas primeiras nove semanas da pandemia, Brasil, 2020. Epidemiologia e Serviços de Saúde, v. 29, 2020.

EJAZ, R. et al. Gender-based incidence, recovery period, and mortality rate of COVID19 among the population of district Attock, Pakistan. Brazilian Journal of Biology, $v$. $83,2021$.

FINSTERER, Josef et al. Peripheral neuropathy in COVID-19 is due to immunemechanisms, pre-existing risk factors, anti-viral drugs, or bedding in the Intensive Care Unit. Arquivos de neuro-psiquiatria, v. 79, p. 924-928, 2021. 
KIVENT. K FORCE. User's Manual. Thessalonique, Greece. 2020. Disponível em: <https://k-invent.com/wp-content/uploads/2020/09/K-FORCmanual.En21_04_20.pdf>. Acesso em: 10 de Dezembro de 2021.

LAKE, Mary A. What we know so far: COVID-19 current clinical knowledge and research. Clinical medicine, v. 20, n. 2, p. 124, 2020.

LOPES, Karolina Rodrigues; CARPES, Marta Fioravanti. Acompanhamento da capacidade funcional de indivíduos infectados pela covid-19: a importante relação entre universidade e sociedade. Anais do Salão Internacional de Ensino, Pesquisa e Extensão, v. 13, n. 2, 2021.

MO, Pingzheng et al. Clinical characteristics of refractory COVID-19 pneumonia in Wuhan, China. Clinical infectious diseases, 2020. Disponível em: <http://dx.doi.org/10.1093/cid/ciaa270>.

NERY, Suellen Belmonte et al. A universidade como rede de apoio e monitoramento de pacientes pós covid-19. Anais do Salão Internacional de Ensino, Pesquisa e Extensão, v. 13, n. 2, 2021.

PIEDRA, Janet Seoane et al. Protocolo de rehabilitación integral para pacientes postCOVID-19. Revista Cubana de Medicina Física y Rehabilitación, v. 12, n. 3, 2020.

PORTO, Natalia Ronquini et al. Funcionalidade de pacientes após infecção por covid19 no município de Londrina-PR. In: Congresso Internacional em Saúde. 2021.

SANTANA, André Vinícius; FONTANA, Andrea Daiane; PITTA, Fabio. Reabilitação pulmonar pós-COVID-19. Jornal brasileiro de Pneumologia, v. 47, 2021.

SILVA, Lidia Cristina Oliveira; PINA, Thaís Anjos; JACÓ, Leina Souza Ormond. Fisioterapia e funcionalidade em pacientes pós covid19: revisão de literatura. Hígiarevista de Ciências da Saúde e Sociais Aplicadas do Oeste Baiano, v. 6, n. 1, 2021. Acesso em 20 de Dezembro de 2021. Disponível em: http://fasb.edu.br/revista/index.php/higia/article/view/637.

SOUZA, Larissa Gonçalves; RANDOW, Raquel; SIVIERO, Pamila Cristina Lima. Reflexões em tempos de COVID-19: diferenciais por sexo e idade. Comunicação em Ciências da Saúde, v. 31, p. 75-83, 2020.

SUN, Tiantian et al. Rehabilitation of patients with COVID-19. Expert Review of Respiratory Medicine, v. 14, n. 12, p. 1249-1256, 2020.

TEIXEIRA, Carmen Fontes de Souza et al. A saúde dos profissionais de saúde no enfrentamento da pandemia de Covid-19. Ciencia \& saude coletiva, v. 25, p. 34653474, 2020.

TENFORDE, Mark W. et al. Symptom duration and risk factors for delayed return to usual health among outpatients with COVID-19 in a multistate health care systems network-United States, March-June 2020. Morbidity and Mortality Weekly Report, v. 69, n. 30 , p. $993,2020$. 
WAN, Suxin et al. Clinical features and treatment of COVID-19 patients in northeast Chongqing. Journal of medical virology, v. 92, n. 7, p. 797-806, 2020.

WANG, Zhuo et al. What are the risk factors of hospital length of stay in the novel coronavirus pneumonia (COVID-19) patients? A survival analysis in southwest China. Plos one, v. 17, n. 1, 2022.

WEINSTOCK-ZLOTNICK, Gwen; MEHTA, Saurabh P. A systematic review of the benefits of occupation-based intervention for patients with upper extremity musculoskeletal disorders. Journal of Hand Therapy, v. 32, n. 2, p. 141-152, 2019.

XIONG, Qiutang et al. Clinical sequelae of COVID-19 survivors in Wuhan, China: a single-centre longitudinal study. Clinical Microbiology and Infection, v. 27, n. 1, p. 89-95, 2021.

\section{Recebido em: 08/03/2022}

Aprovado em: 05/03/2022

Publicado em: 03/02/2022 